\title{
Europe opens new era in synchrotron research
}

Grenoble, France. Future prospects for research into the structure of matter took a major step forward last week with the opening in Grenoble of the 6-GeV European Synchrotron Radiation Facility (ESRF) which extends the wavelength range of synchrotron radiation from 'soft' to 'very hard' X-rays.

The FFr3.6-billion (US\$681-million) ESRF is the first of the so-called thirdgeneration synchrotrons, which produce fine and brilliant beams of X-rays at chosen wavelengths - almost an "X-ray laser", says Yves Petroff, director general of ESRF. The United States will also open a 7-GeV machine (the Advanced Photon Source) at Argonne in 1996-97, and Japan an 8-GeV machine, the SP-8, at Kobe a year later.

Synchrotron radiation was first observed in 1947 by researchers at General Electric in the United States. They found that each time the trajectory of high-energy electrons in a circular accelerator was bent by magnets, the electrons emitted a cone of electromagnetic radiation, similar to the cone of light from the headlights of a car turning a bend.

Initially, scientists saw this synchrotron radiation as simply a curious nuisance that reduced the energy of electrons in particle accelerators. Only in the 1960 s did scientists recognize the potential of synchrotron radiation for overcoming the problems of the conventional Coolidge X-ray tube, which emits relatively feeble and direction-less Xrays that are intense only at a few wavelengths, and cannot be focused or polarized.

First-generation synchrotrons amounted simply to crude X-ray beams clipped on to existing particle accelerators. What sets ESRF apart from previous purpose-built synchrotrons - such as the second-generation 2$\mathrm{GeV}$ machine at the Daresbury Laboratory in Warrington, in the United Kingdom - is its threefold higher energy, the consequent ability to attain wavelengths thousands of times shorter, and the much higher (up to five orders of magnitude) brilliance of its $\mathrm{X}$-ray beams.

ESRF's high energies are obtained by first accelerating electrons to $200 \mathrm{MeV}$ in a linear accelerator, and then to $6 \mathrm{GeV}$ in a

\section{ESRF as 'a model of collaboration'}

Grenoble, France. Built on schedule, within budget and above specification, the European Synchrotron Radiation Facility (ESRF) is a model of how to do big science, according to François Fillon, France's minister of research and higher education.

Fillon would like to see the "pragmatic" ESRF model extended to other big science projects, with different countries contributing according to their scientific and technological needs.

European science, he said at the opening ceremony of the ESRF in Grenoble last week (see above), needs to avoid locking itself into an "overformal-ized institutional approach", which often creates as many problems as it solves.

Aware of Europe's failure to present a united face to its potential partners in the Large Hadron Collider at CERN, the European Laboratory for Particle Physics, Fillon also says that Europe should negotiate en blocon international projects. He would like Europe's science ministers to hold annual meetings on big science projects in order to reach a common position.

One reason for ESRF's success, says Ron Newport, chairman of the ESRF council, is that participants "volunteer" and negotiate their contributions according to "perceived needs and not some predetermined formula".

Yves Petroff, director general of ESRF, says that the security of multi-annual budg- ets is essential. The facilities' members agreed to provide FFr2.2 billion (US $\$ 416$ million) from 1988 until mid-1994 to cover initial construction costs, and a further FFr400 million for construction and FFr1.06 billion for running costs from mid-1994 until 1998.

But in contrast to the United States and Japan, where private companies are planning to pay much of the costs of the proposed third-generation synchrotrons, European companies have no plans to build their own beamlines at ESRF. "We hope this will change", says Petroff.

Despite this lack of corporate funding, Newport points out that European industry need not lose out on benefits from ESRF, as it will still be able to buy time on the facility. ESRF will charge private users FFr3,000 per hour if they publish their results, but will make a surcharge for confidentiality. It will also offer a full contract research service.

FFr1 billion of the FFr2.2 billion costs of the first phase of ESRF construction has already gone into the pockets of European companies, says Newport. In addition, the sophisticated engineering developed at ESRF will be licensed; new optics had to be developed, for example, because conventional optics could not cope with the highpower density of the X-ray beams, namely several kilowatts per square millimetre.

Declan Butler

\author{
IMAGE \\ UNAVAILABLE \\ FOR COPYRIGHT \\ REASONS
}

most an X-ray laser'.

300 -metre-circumference synchrotron. The electrons are then fed into the 844-metrecircumference storage ring (see photo).

Here we find the big third-generation innovation - the addition of 'insertion devices'. Existing synchrotrons produce radiation only when high-energy electrons pass bending magnets in the storage ring.

But ESRF also produces radiation at 29 insertion devices, each of which creates an oscillating magnetic field that forces electrons to slalom about the linear part of their trajectory.

The result is a series of $\mathrm{X}$-ray beamlines that shoot off from the storage ring at tangents, arranged like multiple exits on a motorway roundabout.

Here big science ends, and small science begins. Outside research groups simply take a beam, refine it by passing it through a slit, choose the wavelength they need using a monochromator, and focus the beam onto their sample using a mirror.

Indeed, ESRF will do little in-house research. Four-fifths of user time on public beamlines is reserved for free use by outside research groups. Nine such public beamlines are already installed, and two more are planned by the end of the year; 101 groups have been selected from around 300 applications by peer review for the first trimester of operation.

ESRF has a capacity of 50 beamlines. In addition to the public beamlines, 24 beamlines will also be built and paid for by groups of research institutes - Collaborative Research Groups (CRG) - at a cost of between FFr10 million and FFr20 million each. ESRF will be entitled to hire out onethird of user time on CRG beamlines.

More then 6,000 European researchers now use synchrotron radiation, says Ron Newport, chairman of the ESRF council and head of the Daresbury Laboratory. The list of peer-review committees gives some idea of the range of ESRF's applications - hard condensed matter, soft condensed matter, chemistry, life sciences, surfaces and interfaces, and methods and instrumentation. 